\title{
Targeting Lymphangiogenesis and Lymph Node Metastasis in Liver Cancer
} Sanjukta Chakraborty*

From the Departments of Medical Physiology* and Molecular and Cellular Medicine, ${ }^{\ddagger}$ College of Medicine, Texas A\&M University Health Science Center, Bryan, Texas; the Division of Transplant Surgery, ${ }^{\dagger}$ Department of Surgery, Indiana University School of Medicine, Indianapolis, Indiana; the Division of Gastroenterology and Hepatology, ${ }^{\S}$ Department of Medicine, Indiana University, Indianapolis, Indiana; and the Richard L Roudebush VA Medical Center, ${ }^{\natural}$ Indianapolis, Indiana

Accepted for publication August 26, 2021.

Address correspondence to Sanjukta Chakraborty, Ph.D., Department of Medical Physiology, College of Medicine, Texas A\&M University Health Science Center, 2416 Medical Research Building II, 8447 Riverside Parkway Bryan, TX 77807. E-mail: schakraborty@ tamu.edu.
Increased lymphangiogenesis and lymph node metastasis, the important prognostic indicators of aggressive hepatobiliary malignancies such as hepatocellular cancer and cholangiocarcinoma, are associated with poor patient outcome. The liver produces $25 \%$ to $50 \%$ of total lymphatic fluid in the body and has a dense network of lymphatic vessels. The lymphatic system plays critical roles in fluid homeostasis and inflammation and immune response. Yet, lymphatic vessel alterations and function are grossly understudied in the context of liver pathology. Expansion of the lymphatic network has been documented in clinical samples of liver cancer; and although largely overlooked in the liver, tumor-induced lymphangiogenesis is an important player, increasing tumor metastasis in several cancers. This review aims to provide a detailed perspective on the current knowledge of alterations in the hepatic lymphatic system during liver malignancies, as well as various molecular signaling mechanisms and growth factors that may provide future targets for therapeutic intervention. In addition, the review also addresses current mechanisms and bottlenecks for effective therapeutic targeting of tumor-associated lymphangiogenesis. (Am J Pathol 2021, 191: 2052-2063; https://doi.org/10.1016/j.ajpath.2021.08.011)
With $>780,000$ deaths occurring each year worldwide, liver cancer is the fourth leading cause of cancer-related deaths. ${ }^{1}$ Incidence rates of liver cancers have been increasing in the United States since 1980, with an estimated 42,810 new cases of primary liver cancer and intrahepatic cholangiocarcinoma (iCCA) to be diagnosed in 2020 (30,170 estimated in men, and 12,640 estimated in women) (American Cancer Society, Cancer Statistics Center, http://cancerstatisticscenter.cancer.org, last accessed September 13, 2020). Depending on the cellular origin and anatomic location, liver cancers can be broadly divided into two types: primary, those that start in the liver; and secondary, those that start in another organ and metastasize to the liver. ${ }^{2}$

\section{Types of Liver Cancer}

Depending on the cellular origin and anatomic location, primary liver cancers are as follows:

\section{Hepatocellular Carcinoma}

Hepatocellular carcinoma (HCC) accounts for $>90 \%$ of all primary liver cancers. ${ }^{3}$ It is also a leading cause of death in many parts of the world, especially in low-resource countries. ${ }^{3}$ Several studies looking at prognostic signatures and cellular markers have identified hepatocytes and hepatic progenitor cells as the primary cells of origin of HCC. Risk factors identified for HCC involve: i) nonalcoholic fatty liver disease, caused by metabolic syndromes, such as

\footnotetext{
Supported by Auf-X-Grant Award from Texas A\&M University Health Science Center; Research Enhancement Award, Department of Medical Physiology (S.C.); Cancer Prevention \& Research Institute of Texas grant RP210213 (S.C.); American Heart Association grant 17SDG33670306 (S.C.); Hickam Endowed Chair, Gastroenterology from Indiana University (G.A); a VA Research Senior Career Scientist Award (G.A); and NIH grants DK115184, DK110035, DK076898, DK062975, and AA028711 (S.S.G. and G.A.).

Disclosures: None declared.
} 
obesity and diabetes mellitus, resulting in steatosis, which ultimately causes nonalcoholic steatohepatitis; ii) hepatitis B and $\mathrm{C}$ infection; and iii) excessive alcohol consumption, leading to cirrhosis. Fatty liver disease, diabetes, and alcoholic cirrhosis are also well-documented risk factors for HCC in the United States and Europe. ${ }^{4}$ In addition, genetic diseases, such as $\alpha-1$ antitrypsin deficiency, hemochromatosis, and Wilson disease, can also independently predispose an individual toward $\mathrm{HCC} .^{5}$

\section{Intrahepatic Cholangiocarcinoma}

iCCA, originating in the biliary epithelial cells (ie, cholangiocytes) inside the liver, accounts for $10 \%$ to $20 \%$ of primary liver cancer cases worldwide. It represents the second most common, but the most lethal form of primary liver cancer. Most cases present at late stage of progression, and surgical resection remains the only option for cure, with post-surgery survival time ranging from 27 to 36 months. ${ }^{6}$ Thus, iCCA is considered as one of the most aggressive forms of cancer, with only $15 \%$ of all cholangiocarcinoma (CCA) patients with resectable tumors during diagnosis. Risk factors for CCA are similar to those of HCC. However, biliary inflammation and fibrosis observed in liver diseases, such as primary biliary cholangitis, primary sclerosing cholangitis, and hepatolithiasis, have also been noted to be risk factors for $\mathrm{CCA}{ }^{7,8}$

\section{Hepatic Angiosarcoma}

Primary hepatic angiosarcoma is a relatively rare but aggressive form of liver cancer and represents $0.1 \%$ to $2 \%$ of all primary liver cancers. It is considered the third most common liver malignancy and originates in the endothelial cells lining the blood vessels and lymphatic vessels (LVs) of the liver. ${ }^{9}$ The risk factors for hepatic angiosarcoma are similar to those of HCC and CCA but also include epithelioid hemangioendothelioma, Kaposi sarcoma, and fibrosarcoma. ${ }^{10}$ Because of the lack of specific symptoms and the rarity of cases, the diagnosis of hepatic angiosarcoma remains a challenge. There is a poor prognosis associated with surgical resection, which is the only option for treatment; only $3 \%$ of patients live $>2$ years after surgery, whereas most die within 6 months of diagnosis. ${ }^{11}$

\section{Secondary Liver Cancer}

Cancers originating in other organs metastasize to the liver to form secondary liver cancer. Incidence rates of secondary liver cancers are higher than primary liver cancers in the United States, Europe, Asia, and Africa. ${ }^{2}$

\section{Lymphatic Involvement in Precancerous Liver Disease and Contribution to Disease Progression}

Human liver produces $25 \%$ to $50 \%$ of the body's total lymph fluid. ${ }^{12}$ The LVs of the liver can be divided into two types: intrahepatic LVs, which lack muscle cells; and extrahepatic muscular collecting LVs. ${ }^{13,14}$ Recent evidence from murine models suggests that the lymphatics are organized segmentally, and collecting lymphatics drain to hilar nodes. ${ }^{15}$ Furthermore, the intrahepatic lymphatic vessels are exclusively located near the portal triads. ${ }^{15-17}$ Lymphatic capillaries in the portal tract coalesce into collecting vessels and drain to lymph nodes (LNs) at the hepatic hilum. ${ }^{18}$ Anatomically, hepatic lymphatic system has three distinct locations in the liver: portal, sublobular, and superficial LVs, all originating from hepatic sinusoids. About $80 \%$ of the hepatic lymph is carried by portal LVs, whereas sublobular and superficial LVs carry the rest. An excellent summary of the site of origin and anatomic distribution of the hepatic LVs can be found in the review by Ohtani and Ohtani. ${ }^{13}$ The plasma components of blood filtered by hepatic sinusoidal endothelial cells enter the space of Disse that produces hepatic lymph. This lymph then travels through the space of Mall and is finally carried by the portal LVs. ${ }^{13,19}$ The portal LVs, consisting of interconnecting branches, form a network where the portal tract splits and extends until the terminal portal tract. The sublobular LV is distributed throughout the hepatic sinusoids, space of Disse, and connecting channels between space of Disse and perihepatic interstitial tissue. ${ }^{20}$ As in LNs, the liver provides a space for antigen recognition in interstitial fluid and by immune cells carried by the lymph. The liver also plays a critical role in immune cell trafficking to the lymph nodes via lymphatic fluid. ${ }^{13,14}$ The frequency of lymph node metastasis (LNM) in patients of hepatic malignancy undergoing hepatic resection ranges from $5 \%$ to $50 \%$. $^{21}$ Hence, it is crucial to consider the extent and role of lymphatic dysfunction in precancerous liver diseases. However, these mechanisms are not well characterized, and only a handful of studies have reported lymphatic dysfunction in chronic liver diseases. ${ }^{22}$ Inflammation is one of the prime modulators of cholestatic liver disease and is often associated with viral infection of the liver and drugrelated toxicity. ${ }^{23}$ One of the less common but potentially lethal forms of lymphatic dysfunction occurring after liver injury is fulminant hepatic failure, which is believed to be a manifestation of Hodgkin lymphoma. About $90 \%$ of patients experiencing this devastating condition die before liver transplant. ${ }^{24}$ In fulminant hepatic failure, lymphocytes are hyperactivated and coupled with massive hepatocyte apoptosis and necrosis. In patients with fulminant hepatic failure, coagulopathy and encephalopathy set quickly after the liver insult in the absence of any previous liver condition. An increased lymphatic flow and a large number of 
LVs have also been reported in cirrhosis and hepatic fibrosis. This is maybe due to increased expression of lymphangiogenic growth factors, such as vascular endothelial growth factor (VEGF)-C and VEGF-D during the progression of the pathology. ${ }^{25}$ Increased lymphangiogenesis observed during inflammatory liver diseases has been shown to have a direct correlation with disease severity. ${ }^{24,26}$ In vivo high-resolution fluorescence microscopy in rat models of liver fibrosis and cirrhosis demonstrated that delay of macromolecular blood hepatocytic exchange is associated with increased lymphatic drainage, and lymphatic drainage functions as a compensatory mechanism. $^{24}$

Most cholestatic liver diseases, such as primary sclerosing cholangitis, cirrhosis, and cholelithiasis, are major risk factors for liver cancer development. ${ }^{27}$ Cholestasis is defined as the obstruction of bile flow from the liver. The presence of an inflammatory microenvironment in several liver diseases, such as cirrhosis, fibrosis, primary biliary cholangitis, and primary sclerosing cholangitis, induces infiltration of cytokines such as chemokine (C-C motif) ligand (CCL) 21, by several immune cells, which further causes an increased flow of immune cells to nearby draining $\mathrm{LNs}^{28}$ Given their involvement in a vast range of liver pathologies, lymphatic endothelial cells (LECs) in the liver are believed to participate in different physiological functions. ${ }^{28}$ Levels of lipopolysaccharide are significantly elevated in portal vein circulation during cirrhosis, which induces the expression of NF- $\kappa \mathrm{B}$ in LECs, thus upregulating lymphangiogenesis by increasing Prospero homeobox 1 protein (Prox1) and VEGF receptor (VEGFR)-3 expression. ${ }^{29}$ Cancer cells expressing CCR7 migrate toward CCL21, produced by inflamed LECs, whereas in certain cancers, such as gliomas, CCL21 chemokine gradients drive tumor cell dissemination. LECs also regulate immune evasion, where LECs recruit regulatory $\mathrm{T}$ cells known to cause clonal anergy and death of $\mathrm{CD}^{+} \mathrm{T}$ cells that kill cancer cells. ${ }^{30,31}$ Hence, to identify lymphatic infiltration and early stages of cancer-related lymphangiogenesis, the establishment of molecular markers becomes necessary to precisely recognize lymphangiogenesis in liver.

\section{Lymphatic Markers in Liver Pathologies and Tumor-Related Lymphangiogenesis}

Traditionally, lymphatic markers of LECs have been used to determine lymphatic dysfunction and tumor-related lymphangiogenesis. In the liver, pinpointing a specific lymphatic marker is complicated because several lymphatic markers are expressed by different hepatic and biliary cell types, especially during pathologic conditions. At present, a combination of other markers specific to a pathologic liver condition seems to be the best way for identifying hepatic dysfunction of the lymphatic system. Some of the lymphatic-specific markers that are also expressed by other liver cells are lymphatic vessel hyaluronan receptor 1 (Lyve1), Prox1, podoplanin, and VEGFR-3. An excellent summary of these markers commonly used in other organs along with their expression in different hepatic cell types has been previously provided. ${ }^{16,18}$ Given this complicated scenario, for immunofluorescence analysis and identification of lymphatic infiltration in liver tumors, an effective combination could be the use of one of the lymphatic markers (like LYVE1 or podoplanin) along with a cholangiocyte-specific marker: cytokeratin $19 .{ }^{17}$

\section{Lymphatics and Liver Cancer}

Although the role of lymphatics in hepatic abnormalities remains primarily unexplored, several correlation studies in the two primary liver malignancies, HCC and CCA, have demonstrated that patients with increased LNM and enhanced lymphatic infiltration have significantly poor prognosis and poor patient outcome. Herein, the review summarizes some of the current findings in both HCC and CCA.

\section{Lymphatic Involvement in HCC}

Limited reports in literature provide detailed accounts of lymphatic involvement in HCC. ${ }^{32}$ Furthermore, LNM is a relatively rarer occurrence in HCC. However, several recent studies have found that $\mathrm{LN}$ metastasis was correlated with low survival and aggressive disease progression in $\mathrm{HCC} .^{21,33}$ In a population of HCC patients, worst prognosis was observed in patients with LNM, suggesting that liver resection coupled with lymphadenectomy could have larger patient benefit. ${ }^{34}$ In a study of 403 patients of HCC, regional lymph node metastasis was found in $41 \%$ of patients, whereas periceliac node involvement was observed in $33 \%$ of patients and $23 \%$ had portohepatic lymph node infiltration. ${ }^{35}$ Increased lymphangiogenesis is reported in the intratumoral septa as well as within the bulk of tumor cells in HCC and corelates with significantly lower survival. ${ }^{36} \mathrm{HCC}$ patient samples have increased lymphatic vessels (that are Lyve ${ }^{+-}$ Prox $-1^{+}$), which are pronounced in fibrotic areas surrounding primary tumor. ${ }^{37}$ Cirrhosis, viral hepatitis, and opportunistic infections following orthotopic liver transplantation show enlarged benign lymph nodes in patients. ${ }^{38}$ Because cirrhosis and viral hepatitis are considered to be risk factors for $\mathrm{HCC}$, it is often difficult to assess the main contributing factor behind inflammation and LN remodeling and enlargement observed in such patients. However, the autopsy of HCC patients indicates $27 \%$ to $42 \%$ lymphatic spread. ${ }^{39}$ Three chief prognostic factors of HCC include localized tumor growth, vascular invasion, and LNM. ${ }^{40}$ Furthermore, multiple lymphatic regulators, like hypoxia-inducible factor-1 $\alpha$ and VEGFR-3, have been identified to be biomarkers for HCC LNM $^{41}$ Analysis of serum long noncoding RNA determined three predictive long noncoding RNAs 
(ENST00000418803, long noncoding ZNF35-4:1, and long noncoding EPS15L1-2:1) that could be predictive of the HCC LNM status. ${ }^{42}$ HCC cases that show increased expression of VEGF-C are predisposed to increased LNM. VEGF-D-overexpressing HCC tumor cells promote lymphangiogenesis and lymphatic metastasis and these effects are inhibited in presence of soluble VEGFR-3 that sequestered VEGF-D produced by the tumor cells. ${ }^{4}$ These observations point toward a critical need to address LNM in HCC using lymphatic-targeted molecular therapies.

\section{Lymphatic Involvement in CCA}

CCA or biliary epithelial cancer is a rare but highly aggressive hepatic biliary malignancy, with approximately 5000 new cases being registered each year in the United States alone. ${ }^{43}$ The devastating aggressiveness of CCA is represented by the fact that only $2 \%$ of patients survive 5 years after detection of LNM. ${ }^{44}$ There are three chief types of CCA: iCCA (formerly described), perihilar CCA (identified as the most common type of CCA, representing 50\% to $60 \%$ of all CCA cases), and distal CCA. iCCA originates in the intrahepatic bile ducts close to the liver lymphatics. Perihilar CCA involves tumor development in the origin of left and right hepatic ducts near the hepatic hilum at the junction of the cystic duct and common hepatic duct. ${ }^{45}$ Distal CCA represents $20 \%$ to $30 \%$ of all CCA cases and arises in the cholangiocytes of extrahepatic bile ducts. ${ }^{45}$ Risk factors of CCA are similar to those of HCC and include primary sclerosing cholangitis, cirrhosis, liver fluke infections, hepatitis B virus and hepatitis $\mathrm{C}$ virus infections, choledocholithiasis, and hepatolithiasis, as well as chronic pancreatitis. ${ }^{46}$ Almost all of these risk factors for HCC contribute to the development of an inflammatory microenvironment in the liver, which augments CCA growth and metastasis. ${ }^{43}$ As a result, the CCA tumor microenvironment (TME) is proinflammatory, desmoplastic, and has a dense stroma involving a large number of cell types, such as carcinoma-associated fibroblasts, $\mathrm{T}$ cells, macrophages, and others, which interact closely with CCA cells. ${ }^{47}$ The positive correlation between poor patient prognosis and LNM indicates that tumor-related lymphangiogenesis is a critical determinant of CCA progression. ${ }^{48,49}$ The interaction between carcinoma-associated fibroblasts and CCA is thought to play a role in lymphangiogenesis in the prolymphangiogenic CCA TME ${ }^{47}$ For instance, CCA cells secrete plateletderived growth factor (PDGF)-D, which binds to PDGF receptor- $\beta$ on carcinoma-associated fibroblasts and stimulates carcinoma-associated fibroblasts to secrete VEGF-C. ${ }^{50}$ VEGF-C/VEGFR-3 is up-regulated in the CCA TME and is also related to poor patient prognosis. Hypoxia-inducible factor- $1 \alpha$, which is considered the master regulator of hypoxia-related changes in cancer, supports lymphangiogenesis by promoting expression of angiopoietin (Ang)-1/2, VEGF-C/D, and PDGF-B from neoplastic cells in TME of various cancer types. ${ }^{51}$ Interestingly, the increased expression of hypoxia-inducible factor- $1 \alpha$ in CCA TME has been associated with poor patient prognosis. ${ }^{52}$ Typically, in CCA, high lymphatic vessel density is associated with extensive lymphatic spread and high frequency for CCA recurrence. ${ }^{53}$

\section{Molecular Mechanism of Lymph Node Metastasis in Liver Cancer}

Several molecular pathways have been reported in liver cancers. The following sections highlight some of the growth factors, chemokines, and cytokines that are associated with liver cancer metastasis and tumor-associated lymphangiogenesis.

\section{Growth Factors}

Several growth factors have been implicated, such as the following:

i) Hepatic growth factor: Hepatic growth factor has been shown to instigate proliferation, tube formation, and migration of LECs by downstream extracellular signalregulated kinase $1 / 2$ and phosphatidylinositol 3-kinase signaling. Increased expression of c-Met, the receptor for hepatic growth factor, is associated with poor prognosis and increased tumor spread. ${ }^{54}$

ii) Insulin-like growth factor (IGF): IGF-1/2 are polypeptide hormones and signal through their receptors, IGF-1 receptor/IGF-2 receptor. Known as the IGF system, this group of two growth factors and their respective receptors also includes six IGF binding proteins. Previous literature suggests a prominent role of IGFs in tumorigenesis, cancer proliferation, and survival. ${ }^{55}$ Some of the downstream signaling pathways of IGF include phosphatidylinositol 3-kinase and Akt. In addition, IGF-1/2 may promote lymphangiogenesis through VEGF-C/D pathways as well as induce lymphatic vessel sprouting independent of the VEGFC/D/VEGFR-3 axis. $^{55}$

iii) Fibroblast growth factor (FGF): The FGF family, consisting of structurally similar ligands and receptors (FGF receptors 1, 2, 3, and 4) expressed in endothelial cells, has been known to promote angiogenesis and may also induce lymphangiogenesis either by upregulating Prox 1 or by the Akt/mechanistic target of rapamycin/p70S6K pathway. ${ }^{56,57}$ Increased expression of FGF receptor-2 has been observed in several cancers and is a significant therapeutic target in CCA. ${ }^{58}$ Activating FGF receptor mutations in cancer cells lead to activation of rapidly accelerated fibrosarcoma (RAF)/ mitogen-activated protein kinase kinase 1/extracellular signal-regulated kinase or the AKT/mechanistic target of rapamycin pathways that are known to directly stimulate lymphangiogenesis. $^{59}$ 
iv) Angs: Ang-2, a ligand for endothelial TEK (Tie2) tyrosine kinase receptor, is induced in hypoxic endothelial cells of tumors. ${ }^{60}$ The Ang family consists of well-characterized Ang-1 and Ang-2 with their receptor Tie-2. Limited information is available about Angs in lymphangiogenesis. Ang-1 is shown to play a role in LEC proliferation and lymphatic vessel sprouting, whereas Ang-2 knockout mice exhibited severe lymphatic dysfunction. ${ }^{60}$ Ang-1 plays a role in vessel enlargement and vasculogenesis and also promotes inflammatory lymphangiogenesis. ${ }^{61}$

v) VEGF: Many studies have pointed out the role of the VEGF-C/D/VEGFR-3 axis in cancer progression, in LNM, and associated with poor prognosis. VEGF-C and VEGF-D drive lymphangiogenesis and lymphatic remodeling in tumors that significantly correlate with tumor metastasis to LNs and distant organs. ${ }^{62}$ Notably, lymphatic vessels closer to the metastatic front of the tumor were found to be enlarged and dilated, pointing to the critical regulatory role of VEGFs in mediating tumor-related lymphangiogenesis and LNM.

vi) Endoglin: Endoglin (CD105) is a transmembrane glycoprotein coreceptor of transforming growth factor$\beta$, which was found to be distinctly overexpressed in tumor LECs isolated from metastatic fibrosarcomabased mouse model. The same study showed a similar overexpression of head and neck squamous cell carcinoma and colorectal cancer, thus revealing a possible role for endoglin in the induction of tumor lymphangiogenesis. ${ }^{63}$

vii) Neuropilins: Neuropilins are a family of transmembrane semaphorin and VEGF coreceptors. VEGF-C/D bind to neuropilin-2 and VEGFR-3 to induce lymphangiogenesis. In preclinical studies, antibodies targeting neuropilin-2 have reduced lymphangiogenesis and metastasis. ${ }^{64}$ Thus, in addition to VEGF-C/VEGFR-3, neuropilins can be an essential target for managing tumor lymphangiogenesis.

\section{Inflammatory Chemokines}

Because the TME is predominantly inflammatory in composition, the role of inflammatory chemokines secreted by different cellular members of the TME and cancer cells is fast emerging to be critical in understanding the fundamental concepts of cancer progression, such as increased tumor cell migration and LNM. LECs are critical players in the inflammatory process and secrete several chemokines, such as CCL19 and CCL21, which bind to CCR7, and CXCL12, which binds and signals through $\mathrm{C}-\mathrm{X}-\mathrm{C}$ chemokine receptor type 4 (CXCR4). These chemokines serve essential functions, such as lymphocyte homing and dendritic cell migration to lymphatic vessels. Lymphatics express several chemokines and cytokines as well as many receptors that provide a mechanism of interaction with tumor cells that further contribute to increased LNM. ${ }^{65}$ Tumor cells drive the formation of a prometastatic niche; and in response to inflammation and immune response, $\mathrm{LN}$ LECs show a dramatic response that is in tandem with proliferation of LECs. This further coincides with increased expression of chemokines by the LNs that promote tumor migration. ${ }^{62}$

The overall interaction between the tumor cells and lymphatic endothelial cells that promote tumor growth and lymph node metastasis is schematically outlined in Figure 1.

\section{Role of Lymphatics in Cancer Immunomodulation}

In addition to aberrant lymphangiogenesis during malignancy and pathologic infection, lymphatics play a critical role in regulating some of the vital antitumor responses by immune cells. As the literature is limited about role of lymphatics in immune response of liver cancers, herein we discuss some of the key mechanisms that have been reported for the lymphatics in immune regulation of other cancers. One of the chief molecular factors regulating lymphangiogenesis is $V E G F-C$. In fact, high serum levels of VEGF-C have been suggested as a potential biomarker for determining immunotherapeutic outcome in metastatic melanoma. VEGF-C was shown to potentiate immunotherapy by attracting naïve $\mathrm{T}$ cells, and serum levels of VEGF-C could potentially be a useful biomarker for predicting immunotherapy response. ${ }^{66}$ Murine models lacking dermal LVs lack an adaptive immune response to tumors, thus highlighting the critical role of LVs in initiating antitumor immunity. One way the LVs can mobilize tumor antigens is via dendritic cells transported with lymph to the TME and LNs. ${ }^{30}$ This observation posts a challenge to develop anti-lymphangiogenic therapeutic strategy as blocking LV formation limits adaptive antitumor immunomodulation. In melanoma, LVs are known to recruit $\mathrm{CD}^{+} \mathrm{T}$ cells to the TME via CCL21. The increased presence of activated CD8 ${ }^{+} \mathrm{T}$ cells has been found in primary melanoma tumor sites thought to occur via VEGF-C/VEGFR-3 signaling. ${ }^{67}$ Contrary to the positive effects of lymphatics in cancer immunotherapy, LVs are also known to carry immunosuppressive factors from solid tumors, thus compromising antitumor immunity. ${ }^{68}$ Some of the antigens transported via lymph and scavenged and presented by LECs are used by cancer cells to develop tolerance against $\mathrm{CD} 8^{+} \mathrm{T}$ cells. ${ }^{69}$ LECs show several similarities to liver sinusoidal endothelial cells with regard to antigen processing and cross-presenting capacity. However, they do not show similar immunostimulatory phenotype, as exhibited by the liver sinusoidal endothelial cells (SLECs), and have low expression of costimulatory molecules, such as CD40, CD80, and CD86. This has been attributed to different levels of endotoxin in the portal blood compared with peripheral lymph. ${ }^{69}$ LECs are also known to 


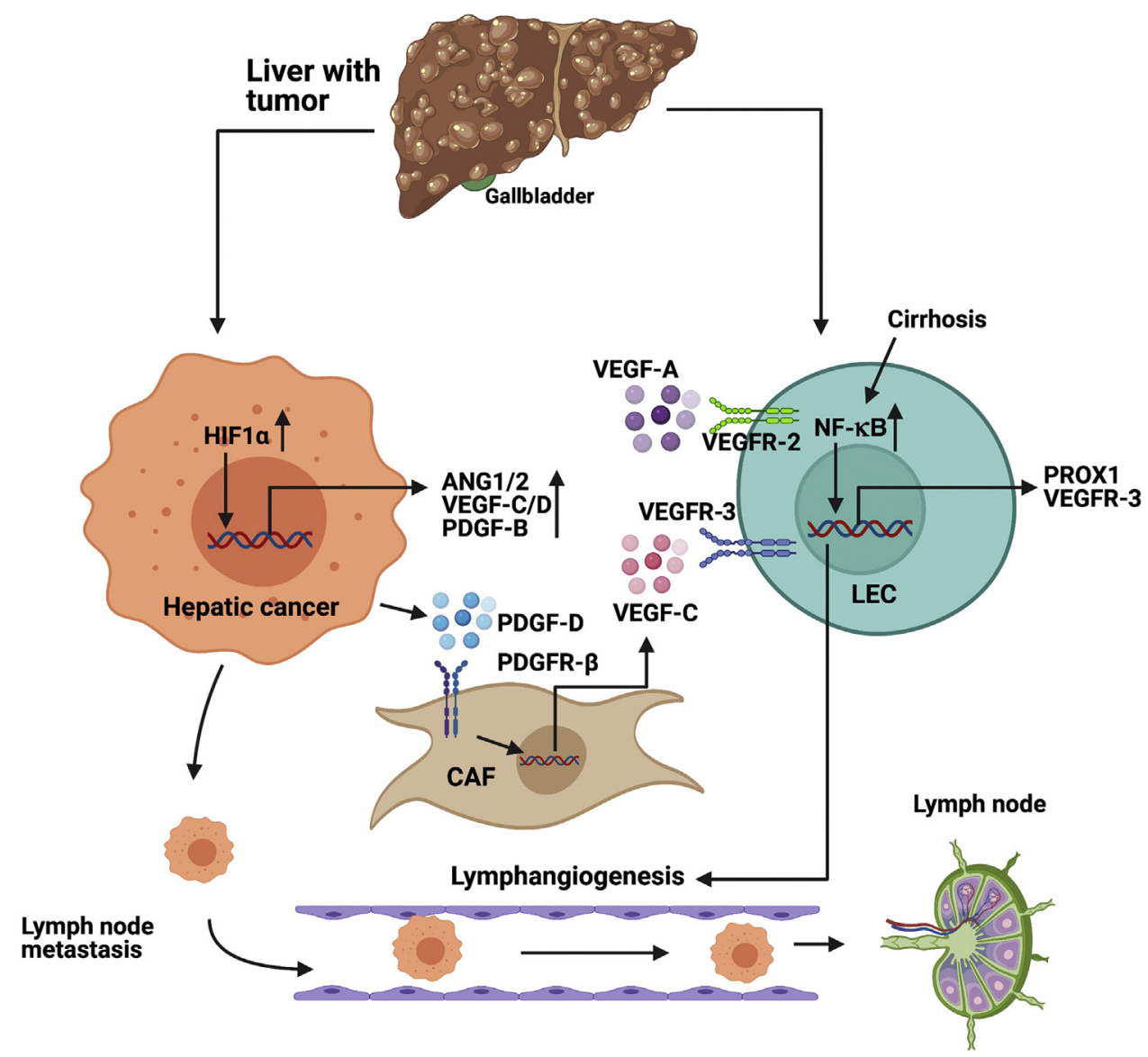

Figure 1 Molecular pathways that promote lymphangiogenesis in liver cancers. Under hypoxia, hypoxia-inducible factor-1 $\alpha$ (HIF1 $\alpha$ ) increases the transcription of lymphangiogenic factors, such as angiopoietin-1/2 (ANG1/2), vascular endothelial growth factor (VEGF)-C/D, platelet-derived growth factor (PDGF)-B, and PDGF-D. PDGF-D secreted from hepatic cancer cells bind to PDGF receptor (PDGFR)-B expressed on the surface of cancer-associated fibroblast (CAFs), which, in turn, leads to increased transcription of VEGF-C from CAFs. The VEGF-C secreted from CAFs binds to VEGF receptor (VEGFR)-3 on lymphatic endothelial cells (LECS) and induces lymphangiogenesis. In the background of preexisting liver diseases, such as cirrhosis, NF- $\kappa B$ pathway is stimulated in LECs, which increases transcription of Prospero homeobox 1 protein (PROX1) and VEGFR-3, thus up-regulating LEC proliferation and lymphangiogenesis. All these processes lead to a high degree of lymphangiogenesis in the hepatic tumor microenvironment, ultimately aggravating lymph node metastasis. Generated with BioRender.com (Toronto, ON, Canada).

present programmed death-ligand 1 to $\mathrm{CD}^{+} \mathrm{T}$ cells to cause T-cell clonal anergy. ${ }^{70}$

\section{Targets and Bottlenecks in Therapeutic Targeting of Lymphatics and Lymphangiogenesis in Liver Cancer}

Therapies targeting tumor-related neovascularization over the past two decades have mainly focused on angiogenesis. Currently, $>20$ different anti-angiogenic drugs exist that have been approved by the Food and Drug Administration of the United States. ${ }^{71}$ However, despite this growing number of anti-angiogenic drug discoveries, the impact of these drugs on cancer patient health has been modest at best, with no existing drugs targeting lymphangiogenesis. Furthermore, the mechanisms of lymphangiogenesis remain even more poorly understood in the context of liver cancers, further challenging development of effective therapies. ${ }^{46}$ Because of the grim outcome after LNM in cancer patients, there remains a critical need to understand the specific molecular mechanisms of neovascularization while considering tumor-related lymphangiogenesis. In most of these cancer types, tumor-related lymphangiogenesis involves high levels of VEGF-C production. VEGF-C, which signals through VEGFR-2/3, induces LEC division, survival, and LV formation. ${ }^{72}$ Studies targeting tumor lymphangiogenesis in mouse have had mixed success. These studies show that although tumor cells exploit lymphatic drainage to hijack normal immune cell trafficking and reach $\mathrm{LN}$, antitumor $\mathrm{CD}^{+} \mathrm{T}$ cells also need lymphatic drainage for active antitumor surveillance, which may explain the minimal efficacy of this study. ${ }^{73}$ A recent clinical study targeting VEGFR-3 was developed, which did not show heightened reduction of tumor growth. ${ }^{74}$ Tumor-related lymphangiogenesis can occur in distant organs and LNs before LNM, due to preconditioning by primary tumor cells to form a prometastatic niche. ${ }^{62}$ Gogineni et $\mathrm{al}^{75}$ showed that lymphatics of distant organs undergo significant structural 
remodeling that is affected by the stage of cancer progression. Post-LNM targeting VEGF-C with blocking antibodies inhibited lung metastasis in murine cancer model. ${ }^{75}$ Postnatal lymphangiogenesis, however, is not limited to only VEGF-C, and cytokines, growth factors, and hormones have been shown to induce VEGFR-3-independent lymphangiogenesis as well. ${ }^{76}$ The quest for a suitable, robust lymphangiogenic target is further complicated by active molecular cross talk between blood and LECs. VEGFR-2, expressed on blood and lymphatic endothelium, and VEGFR-3, expressed on LECs and tumor-related blood vessels, are two examples of angiogenic and lymphangiogenic regulator overlap. ${ }^{77}$ Interestingly, it has also been observed that LNM can occur independent of lymphangiogenesis, where cancer cells enter old LVs rather than producing new ones. ${ }^{78}$ Critical studies highlighting the role of tumor-associated macrophages (TAMs) have shown that VEGFR-3 ${ }^{+}$TAMs produce VEGF$\mathrm{C}$, leading to lymphangiogenesis and ensuing therapeutic resistance following administration of paclitaxel in breast and lung cancer patients. ${ }^{79}$ In murine models of breast cancer podoplanin $^{+}$TAMs in close apposition to lymphatic vessels via $\beta 4$-integrin and laminin 5 interaction produced transforming growth factor- $\beta 1$ that resulted in stronger attachment of TAMs to LVs and remodeled LVs to increase LNM. ${ }^{80}$ Multiple routes of tumor dissemination from sentinel lymph nodes (SLNs) exist. Cancer cells enter blood vessels preexisting in sentinel LNs, and from there spread to distant organs, which suggests that lymphangiogenic targeting of distant tissues could be more promising than targeting lymphangiogenesis in SLNs. ${ }^{81,82}$

Thus, a deeper understanding of molecular mechanisms of tumor-related lymphangiogenesis and LNM, coupled with patient-specific TME changes in liver cancers, is needed to develop anti-lymphangiogenic molecular targets that can inhibit LNM. The following section discusses some of the present advances and therapeutics that target increased tumor vasculature in hepatobiliary cancers. These include the following categories.

\section{Drugs Targeting Vasculogenesis in Liver Cancer}

In HCC, which is characterized by robust angiogenesis in the TME, the standard treatment regimen of transcatheter chemoembolization chemotherapy has yielded limited success because of intratumoral heterogeneity and the role of chronic liver diseases that often accompany HCC in affecting liver parenchyma. Hence, with the approval of sorafenib, a multitargeting tyrosine kinase inhibitor, the focus of treatment has shifted to targeting vasculogenesis in HCC. Different TME cells, including heterogeneous cancer cells, macrophages, and endothelial cells, are regulated by the VEGF signaling pathway in HCC. ${ }^{83}$ Currently, antiangiogenic systemic therapies in clinical trials in the United States, European Union, and China target VEGF and the corresponding receptors. In addition to VEGF, multitargeting tyrosine kinase inhibitors such as the PDGF, FGF, angiopoietin, and endoglin also target signaling pathways. Some examples include the following:

i) Sorafenib: Sorafenib is an oral multikinase inhibitor that was approved in 2008 to treat inoperable HCC. It targets VEGF receptors (VEGFR-2/3), RAF proto-oncogene, serine/threonine kinase (Raf-1), and PDGF receptor- $\beta$, thus blocking tumor cell proliferation. In spite of different adverse effects, such as hand-foot syndrome, hypertension, and diarrhea, associated with sorafenib therapy, there has been significant progress in effectively treating these conditions. Hence, in response to this therapy, the overall survival of advanced-stage HCC patients has increased significantly in both Asian (from 6.5 to 8.5 to 8.9 months) and non-Asian (from 10.7 to 11.8 to 15.1 months) groups, between 2005 and 2007. ${ }^{84}$ Because of improvement of overall survival and higher tolerance to adverse effects, sorafenib remains to be a first-line drug in treating HCC. Because VEGF-C and RAF signaling are important regulators of tumor-related lymphangiogenesis, sorafenib could also potentially suppress lymphangiogenesis in liver cancers. Currently, the effects of sorafenib in reducing lymphatic vessel density in liver cancer patients remains unexplored. However, one drawback of such tyrosine kinase inhibitor is the structural similarity among multiple tyrosine kinase receptors, thus making it difficult to target a specific pathway. Interestingly, a study conducted with keratoplastic mice showed reduction of total lymphangiogenesis, VEGF-C, and VEGFR-3 expression compared with control group. ${ }^{85}$ Thus, it may be of significance to explore the role of sorafenib in targeting lymphangiogenesis in liver cancer as well.

ii) Lenvatinib (E7080): Lenvatinib is another tyrosine kinase inhibitor that targets VEGFRs (1-3), PDGF receptor, and FGF receptor. A randomized, open-label, noninferiority designed trial led to its approval in 2018, as a first-line treatment of patients with unresectable HCC. ${ }^{86}$ Lenvatinib inhibits LNM of MDA-MB-231 (human breast cancer cell line) via VEGFR-3 inhibition. In mouse xenograft model of breast cancer with lenvatinib-treated MDA-MB231 cells, there was a significant reduction in lymphatic vessel density, regional LNM, and lung metastasis. In addition, lenvatinib also significantly decreased lymphatic vessel density within metastatic nodules at LNs after primary tumor resection. ${ }^{87}$ Furthermore, it inhibits VEGF- and FGF-driven proliferation and angiogenic mechanisms. ${ }^{88}$

\section{Antibodies and Decoy Receptors}

Because of the importance of the VEGF-C/VEGFR-3 signaling axis in tumor-related lymphangiogenesis, many studies have explored the application of decoy receptors. These decoy receptors are soluble forms of VEGFR-3 that bind to and trap available VEGF-C. For example, He et al ${ }^{89}$ showed that adenoviral vector-mediated systemic delivery of soluble VEGFR-3-IgG significantly reduced macrometastasis and the population of proliferating LECs. 


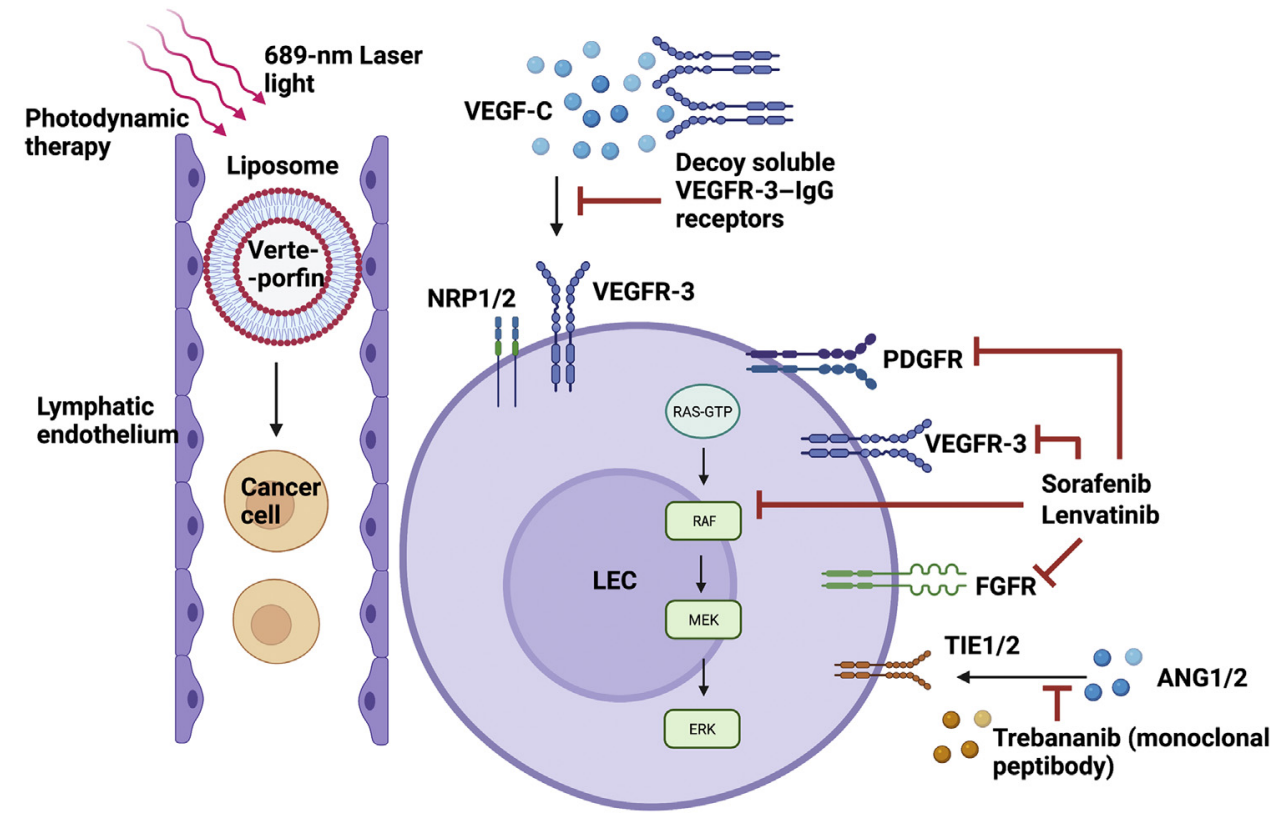

Figure 2 Current therapeutic and treatment modalities targeting tumor lymphangiogenesis. Soluble vascular endothelial growth factor receptor (VEGFR)-3 receptors serve as decoy agents that bind and inhibit soluble vascular endothelial growth factor (VEGF)-C, thus keeping them away from VEGFR-3 or neuropilin1/2 (NRP1/2) coreceptor on lymphatic endothelial cell (LEC) surface. Multikinase inhibitors, like sorafenib and lenvatinib, bind to and block multiple receptors (which bind to lymphangiogenic factors), such as platelet-derived growth factor receptor (PDGFR), VEGFR-3, fibroblast growth factor receptor (FGFR), and rapidly accelerated fibrosarcoma (RAF) kinases. Monoclonal peptibodies, like trebananib, bind to tyrosine kinase with Ig-like and epidermal growth factor (EGF)-like domains 1/2 (TIE1/2) receptor, thus preventing binding of angiopoietin-1/2 (ANG1/2). Photodynamic therapy takes place through liposomemediated, lymphatic-specific delivery of verteporfin, which can kill cancer cells inside lymphatic vessels (LVs), thus reducing cancer metastasis. This specifically happens only inside the LVs. MEK, mitogen-activated protein kinase kinase; RAS, rat sarcoma. Generated with BioRender.com (Toronto, ON, Canada).

Furthermore, sentinel lymph node metastasis was decreased in tumor-bearing mice in a different study, where tumor cells expressing soluble VEGFR-3-Ig blocked VEGF-C while reducing the formation of tumor lymphatics. ${ }^{90}$

\section{Monoclonal Antibodies}

In addition to decoy receptors, monoclonal antibodies blocking VEGFR-3 have also been used to target lymphangiogenesis. Bevacizumab is one such anti-VEGF antibody being tested in colon, kidney, and non-small-cell lung cancer treatment. The combinatorial therapy of bevacizumab with VGX-100 (which is an anti-VEGF-C antibody) has shown considerable efficacy. However, one of the limitations of anti-VEGF-C therapies is the existence of other signaling pathways activating lymphangiogenesis. Another peptibody targeting lymphangiogenic or angiogenic factors is trebananib (AMG-386), which is an angiopoietin-1/2 neutralizing agent. Trebananib consists of Ang-1/2 binding sequence and $\mathrm{Fc}$ region of Ang-1/2 antibody. By preventing Tie- 2 receptor binding by Ang-1/2, tumor growth has been shown to reduce in mouse xenograft models. ${ }^{91}$ In addition to targeting VEGF-C/VEGFR-3, VEGFR-2 has also been shown to play an important role in lymphangiogenesis. Ramucirumab, a human monoclonal anti-VEGFR-2 antibody, may be important in targeting VEGFR-2-mediated lymphangiogenesis. ${ }^{92}$

\section{Photodynamic Therapy}

Photodynamic therapeutic regimens have shown promising results for liver malignancies, such as CCA, that often present at inoperable stages. ${ }^{93}$ Photodynamic therapy involves using visible light of a specific wavelength and has been used to target metastatic cancer cells in transit through LVs. Tammela et $\mathrm{al}^{94}$ used liposome-mediated verteporfin delivery to LVs, which caused a $37.5 \%$ reduction of melanoma metastasis in the ears of a mouse melanoma model. One crucial difference between VEGFR-3 inhibition-based therapies and photodynamic therapy is the specific reduction of preexisting lymphatic vessels, whereas with decoy receptors and antibodies, only new lymphatic growth can be targeted. Lymphatic ablation occured at low dose of verteporfin ( $25 \mathrm{ng}$ ); however, the blood vessels were not affected At a higher dose (100 ng) of verteporfin, local blood vessels were affected as well. Thus, with photodynamic therapy, it is possible to perform site-specific lymphatic ablation, which, in a dose- and intensity-dependent manner, can be used to target lymphatic versus blood vessels. ${ }^{95}$

The different strategies and therapies used to target various facets of LEC activation or metastatic target cells in hepatobiliary malignancies are shown in Figure 2. 


\section{Molecular Mechanisms of Therapeutic Resistance in Liver Cancer}

One critical limitation to primary liver cancer management is that it is often completely nonresponsive to traditional pharmacologic treatments. Several experimental models are currently in use for developing a better understanding of drug resistance in liver cancers. These include both in vitro culture-based models, multicellular three-dimensional models (spheroids and organoids) ${ }^{96}$ that mimic the TME, and several in vivo orthotopic mouse tumor xenografts or chemically or genetically induced liver carcinogenesis in mouse models. These are extensively reviewed by Marin et al. ${ }^{97}$ Critically important regulators of therapeutic resistance in liver cancers are the ATP-binding cassette (ABC) transporters, such as multidrug resistance protein 1/P-glycoprotein (ABCB1), multi-drug resistance protein 2 (MRP2; ABCC2) in hepatoblastoma and $\mathrm{HCC}$, and MRP3 (ABCC3) in CCA. ${ }^{98}$ Plasma membrane vesicles used in several assays have revealed important information regarding drug development in liver cancers. One such assay is the ATPase assay, which uses inside-out plasma membrane vesicles as well as leaky plasma membrane vesicles to determine if a potential drug candidate can serve as a substrate and/or inhibitor. ${ }^{99}$ Mechanisms of chemoresistance (MOC) have been recently established in CCA, which are as follows: decreased drug uptake, leading to changes in intracellular circulating drug (MOC-1a); enhanced drug efflux (MOC-1b); altered relative concentration of prodrug, active drug, and inactive metabolites (MOC-2); changes in the molecular targets of antitumor drugs (MOC3); enhanced DNA damage repair due to drug in tumor cells (MOC-4); and impaired apoptosis, leading to prolonged tumor cell survival (MOC-5). ${ }^{100}$ Although these mechanisms have been studied in liver cancer cells, to develop anti-lymphangiogenic drugs, the role of these molecular resistance mechanisms in LECs, TAMs, or various other cellular TME members also needs to be explored.

\section{Conclusion and Future Directions}

The role of lymphatics in the context of chronic and acute liver diseases, including hepatic cancers, has been grossly understudied. The poor patient outcome associated with enhanced lymphatic infiltration in different hepatobiliary malignancies, as highlighted above, warrants studies dedicated to understanding the following: i) identification of robust LEC markers for liver, ii) determination of the specific role of LECs in progression of various liver disease, and iii) identification of molecular lymphatic targets for improved therapeutic outcomes.

i) Identification of robust LEC markers for the liver: Currently, the known molecular markers of LECs are expressed by one or more hepatic cell types, such as cholangiocytes, hepatocytes, or liver sinusoidal endothelial cells. Therefore, a combination of markers has been used in some recent studies concerning hepatic cell type and disease variant. For instance, podoplanin/ Lyve1 and cytokeratin 19 have been used to distinguish LECs and cholangiocytes. ${ }^{16}$

ii) Determination of the specific role of LECs in the progression of various liver diseases: Up-regulation of different molecular factors, such as VEGF-C, multiple cytokines, and chemokines, has been correlated with increased LNM and poor patient prognosis in other cancer types. However, the role of these molecular factors in the context of LEC involvement in hepatic cancers needs to be primarily evaluated to unravel new interaction pathways that govern the cross talk of LEC and primary/secondary hepatic cancer cells. Some of these molecular cues can be derived from the proteins responsible for inducing an inflammatory stroma, which also increases lymphangiogenesis.

iii) Identification of molecular lymphatic targets for improved therapeutic outcomes: The involvement of lymphatics in shaping the chemotherapeutic outcomes of hepatic cancers is of high clinical significance due to patients' poor prognosis with LNM. Because programmed death-ligand 1-bearing LECs modulate immunologic responses to cancer, identifying new molecular targets can help improve the response to techniques, like cancer immunotherapy. Furthermore, most traditional therapies preclude the lymphatics, thereby keeping an active channel of metastasis.

To develop targeted therapeutic approaches for LNM, tumor-related lymphangiogenesis, and immune alteration by lymphatics, the necessary evil of LV formation during cancer must be addressed. Therefore, future studies with a greater attention to the area of lymphatic involvement in liver malignancy are in dire need.

\section{Author Contributions}

S.R., P.B., and S.C. performed literature search and drafted the manuscript; S.R. prepared the figures; S.R., P.B., B.E., K.J.B., D.Z., G.A., S.G., and S.C. edited, critically revised, and proofread the manuscript; all authors read and approved the final manuscript.

\section{References}

1. Dasgupta P, Henshaw C, Youlden DR, Clark PJ, Aitken JF, Baade PD: Global trends in incidence rates of primary adult liver cancers: a systematic review and meta-analysis. Front Oncol 2020, 10:171

2. Ananthakrishnan A, Gogineni V, Saeian K: Epidemiology of primary and secondary liver cancers. Semin Intervent Radiol 2006, 23:47-63

3. Yang JD, Hainaut P, Gores GJ, Amadou A, Plymoth A, Roberts LR: A global view of hepatocellular carcinoma: trends, risk, prevention and management. Nat Rev Gastroenterol Hepatol 2019, 16:589-604

4. Thelen A, Scholz A, Benckert C, von Marschall Z, Schroder M, Wiedenmann B, Neuhaus P, Rosewicz S, Jonas S: VEGF-D promotes 
tumor growth and lymphatic spread in a mouse model of hepatocellular carcinoma. Int J Cancer 2008, 122:2471-2481

5. McGlynn KA, London WT: The global epidemiology of hepatocellular carcinoma: present and future. Clin Liver Dis 2011, 15: 223-243, vii-x

6. Esnaola NF, Meyer JE, Karachristos A, Maranki JL, Camp ER, Denlinger CS: Evaluation and management of intrahepatic and extrahepatic cholangiocarcinoma. Cancer 2016, 122:1349-1369

7. Nakeeb A, Pitt HA, Sohn TA, Coleman J, Abrams RA, Piantadosi S, Hruban RH, Lillemoe KD, Yeo CJ, Cameron JL: Cholangiocarcinoma: a spectrum of intrahepatic, perihilar, and distal tumors. Ann Surg 1996, 224:463-473; discussion 473-475

8. Amini N, Ejaz A, Spolverato G, Kim Y, Herman JM, Pawlik TM: Temporal trends in liver-directed therapy of patients with intrahepatic cholangiocarcinoma in the United States: a population-based analysis. J Surg Oncol 2014, 110:163-170

9. Millan M, Delgado A, Caicedo LA, Arrunategui AM, Meneses CA, Villegas JI, Serrano O, Caicedo L, Duque M, Echeverri GJ: Liver angiosarcoma: rare tumour associated with a poor prognosis, literature review and case report. Int J Surg Case Rep 2016, 28:165-168

10. Chaudhary P, Bhadana U, Singh RA, Ahuja A: Primary hepatic angiosarcoma. Eur J Surg Oncol 2015, 41:1137-1143

11. Yi LL, Zhang JX, Zhou SG, Wang J, Huang YQ, Li J, Yu X, Wang RN: CT and MRI studies of hepatic angiosarcoma. Clin Radiol 2019, 74:406.e1-e8

12. Barrowman JA: Hepatic lymph and lymphatics. Edited by McIntyre N, Benhamou JP, Bircher J, Rizzetto M. In Oxford Textbook of Clinical Hepatology. New York, NY: Oxford University Press, 1991. pp. 37-40

13. Ohtani O, Ohtani Y: Lymph circulation in the liver. Anat Rec (Hoboken) 2008, 291:643-652

14. Tanaka M, Iwakiri Y: Lymphatics in the liver. Curr Opin Immunol 2018, 53:137-142

15. Frenkel NC, Poghosyan S, Verheem A, Padera TP, Rinkes I, Kranenburg O, Hagendoorn J: Liver lymphatic drainage patterns follow segmental anatomy in a murine model. Sci Rep 2020, 10: 21808

16. Burchill MA, Goldberg AR, Tamburini BAJ: Emerging roles for lymphatics in chronic liver disease. Front Physiol 2019, 10:1579

17. Trutmann M, Sasse D: The lymphatics of the liver. Anat Embryol (Berl) 1994, 190:201-209

18. Tanaka M, Iwakiri Y: The hepatic lymphatic vascular system: structure, function, markers, and lymphangiogenesis. Cell Mol Gastroenterol Hepatol 2016, 2:733-749

19. Iwakiri Y: The lymphatic system: a new frontier in hepatology. Hepatology 2016, 64:706-707

20. Poonkhum R, Pisetpaisan K, Wang BJ, Anupunpisit V, Ohtani Y, Ohtani O: Origins and pathways of fluid entering sublobular lymphatic vessels in cat livers. Arch Histol Cytol 2003, 66: $317-326$

21. Amini N, Ejaz A, Spolverato G, Maithel SK, Kim Y, Pawlik TM: Management of lymph nodes during resection of hepatocellular carcinoma and intrahepatic cholangiocarcinoma: a systematic review. J Gastrointest Surg 2014, 18:2136-2148

22. Chaudhry S, Emond J, Griesemer A: Immune cell trafficking to the liver. Transplantation 2019, 103:1323-1337

23. Delemos AS, Friedman LS: Systemic causes of cholestasis. Clin Liver Dis 2013, 17:301-317

24. Yokomori H, Oda M, Kaneko F, Kawachi S, Tanabe M, Yoshimura K, Kitagawa Y, Hibi T: Lymphatic marker podoplanin/D2-40 in human advanced cirrhotic liver-re-evaluations of microlymphatic abnormalities. BMC Gastroenterol 2010, $10: 131$

25. Franchitto A, Onori P, Renzi A, Carpino G, Mancinelli R, Alvaro D, Gaudio E: Expression of vascular endothelial growth factors and their receptors by hepatic progenitor cells in human liver diseases. Hepatobiliary Surg Nutr 2013, 2:68-77
26. Corpechot C, Carrat F, Poupon R, Poupon RE: Primary biliary cirrhosis: incidence and predictive factors of cirrhosis development in ursodiol-treated patients. Gastroenterology 2002, 122:652-658

27. Razumilava N, Gores GJ, Lindor KD: Cancer surveillance in patients with primary sclerosing cholangitis. Hepatology 2011, 54: $1842-1852$

28. Lukacs-Kornek V: The role of lymphatic endothelial cells in liver injury and tumor development. Front Immunol 2016, 7:548

29. Flister MJ, Wilber A, Hall KL, Iwata C, Miyazono K, Nisato RE, Pepper MS, Zawieja DC, Ran S: Inflammation induces lymphangiogenesis through up-regulation of VEGFR-3 mediated by NFkappaB and Prox1. Blood 2010, 115:418-429

30. Lund AW, Wagner M, Fankhauser M, Steinskog ES, Broggi MA, Spranger S, Gajewski TF, Alitalo K, Eikesdal HP, Wiig H, Swartz MA: Lymphatic vessels regulate immune microenvironments in human and murine melanoma. J Clin Invest 2016, 126:3389-3402

31. Swartz MA: Immunomodulatory roles of lymphatic vessels in cancer progression. Cancer Immunol Res 2014, 2:701-707

32. Xiaohong S, Huikai L, Feng W, Ti Z, Yunlong C, Qiang L: Clinical significance of lymph node metastasis in patients undergoing partial hepatectomy for hepatocellular carcinoma. World J Surg 2010, 34: $1028-1033$

33. Xia F, Wu L, Lau WY, Li G, Huan H, Qian C, Ma K, Bie P: Positive lymph node metastasis has a marked impact on the long-term survival of patients with hepatocellular carcinoma with extrahepatic metastasis. PLoS One 2014, 9:e95889

34. Lee CW, Chan KM, Lee CF, Yu MC, Lee WC, Wu TJ, Chen MF: Hepatic resection for hepatocellular carcinoma with lymph node metastasis: clinicopathological analysis and survival outcome. Asian J Surg 2011, 34:53-62

35. Katyal S, Oliver JH 3rd, Peterson MS, Ferris JV, Carr BS, Baron RL: Extrahepatic metastases of hepatocellular carcinoma. Radiology 2000, 216:698-703

36. Thelen A, Jonas S, Benckert C, Weichert W, Schott E, Botcher C, Dietz E, Wiedenmann B, Neuhaus P, Scholz A: Tumor-associated lymphangiogenesis correlates with prognosis after resection of human hepatocellular carcinoma. Ann Surg Oncol 2009, 16:1222-1230

37. Mouta Carreira C, Nasser SM, di Tomaso E, Padera TP, Boucher Y, Tomarev SI, Jain RK: LYVE-1 is not restricted to the lymph vessels: expression in normal liver blood sinusoids and down-regulation in human liver cancer and cirrhosis. Cancer Res 2001, 61:8079-8084

38. Dodd GD 3rd, Baron RL, Oliver JH 3rd, Federle MP, Baumgartel PB: Enlarged abdominal lymph nodes in end-stage cirrhosis: CThistopathologic correlation in 507 patients. Radiology 1997, 203:127-130

39. Nakashima T, Kojiro M: [Pathology of hepatocellular carcinoma]. Japanese. Nihon Geka Gakkai Zasshi 1983, 84:939-942

40. Qin LX, Tang ZY: The prognostic molecular markers in hepatocellular carcinoma. World J Gastroenterol 2002, 8:385-392

41. Xiang ZL, Zeng ZC, Tang ZY, Fan J, He J, Zeng HY, Zhu XD: Potential prognostic biomarkers for bone metastasis from hepatocellular carcinoma. Oncologist 2011, 16:1028-1039

42. Ma J, Zhang L, Bian HR, Lu ZG, Zhu L, Yang P, Zeng ZC, Xiang ZL: A noninvasive prediction nomogram for lymph node metastasis of hepatocellular carcinoma based on serum long noncoding RNAs. Biomed Res Int 2019, 2019:1710670

43. Banales JM, Marin JJG, Lamarca A, Rodrigues PM, Khan SA, Roberts LR, Cardinale V, Carpino G, Andersen JB, Braconi C, Calvisi DF, Perugorria MJ, Fabris L, Boulter L, Macias RIR, Gaudio E, Alvaro D, Gradilone SA, Strazzabosco M, Marzioni M, Coulouarn C, Fouassier L, Raggi C, Invernizzi P, Mertens JC, Moncsek A, Rizvi S, Heimbach J, Koerkamp BG, Bruix J, Forner A, Bridgewater J, Valle JW, Gores GJ: Cholangiocarcinoma 2020: the next horizon in mechanisms and management. Nat Rev Gastroenterol Hepatol 2020, 17:557-588

44. Banales JM, Cardinale V, Carpino G, Marzioni M, Andersen JB, Invernizzi P, Lind GE, Folseraas T, Forbes SJ, Fouassier L, Geier A, Calvisi DF, Mertens JC, Trauner M, Benedetti A, Maroni L, Vaquero J, 
Macias RI, Raggi C, Perugorria MJ, Gaudio E, Boberg KM, Marin JJ, Alvaro D: Expert consensus document: cholangiocarcinoma: current knowledge and future perspectives consensus statement from the European Network for the Study of Cholangiocarcinoma (ENS-CCA). Nat Rev Gastroenterol Hepatol 2016, 13:261-280

45. Rizvi S, Gores GJ: Pathogenesis, diagnosis, and management of cholangiocarcinoma. Gastroenterology 2013, 145:1215-1229

46. Roy S, Glaser S, Chakraborty S: Inflammation and progression of cholangiocarcinoma: role of angiogenic and lymphangiogenic mechanisms. Front Med (Lausanne) 2019, 6:293

47. Cadamuro M, Morton SD, Strazzabosco M, Fabris L: Unveiling the role of tumor reactive stroma in cholangiocarcinoma: an opportunity for new therapeutic strategies. Transl Gastrointest Cancer 2013, 2: $130-144$

48. Fabris L, Alvaro D: The prognosis of perihilar cholangiocarcinoma after radical treatments. Hepatology 2012, 56:800-802

49. Sha M, Jeong S, Wang X, Tong Y, Cao J, Sun HY, Xia L, Xu N, Xi ZF, Zhang JJ, Kong XN, Xia Q: Tumor-associated lymphangiogenesis predicts unfavorable prognosis of intrahepatic cholangiocarcinoma. BMC Cancer 2019, 19:208

50. Cadamuro M, Brivio S, Mertens J, Vismara M, Moncsek A, Milani C, Fingas C, Cristina Malerba M, Nardo G, Dall'Olmo L, Milani E, Mariotti V, Stecca T, Massani M, Spirli C, Fiorotto R, Indraccolo S, Strazzabosco M, Fabris L: Platelet-derived growth factor-D enables liver myofibroblasts to promote tumor lymphangiogenesis in cholangiocarcinoma. J Hepatol 2019, 70:700-709

51. Roy S, Kumaravel S, Sharma A, Duran CL, Bayless KJ, Chakraborty S: Hypoxic tumor microenvironment: implications for cancer therapy. Exp Biol Med (Maywood) 2020, 245:1073-1086

52. Thongchot S, Yongvanit P, Loilome W, Seubwai W, Phunicom K, Tassaneeyakul W, Pairojkul C, Promkotra W, Techasen A, Namwat N: High expression of HIF-1alpha, BNIP3 and PI3KC3: hypoxia-induced autophagy predicts cholangiocarcinoma survival and metastasis. Asian Pac J Cancer Prev 2014, 15:5873-5878

53. Thelen A, Scholz A, Benckert C, Schroder M, Weichert W, Wiedenmann B, Neuhaus P, Jonas S: Microvessel density correlates with lymph node metastases and prognosis in hilar cholangiocarcinoma. J Gastroenterol 2008, 43:959-966

54. Organ SL, Tsao MS: An overview of the c-MET signaling pathway. Ther Adv Med Oncol 2011, 3:S7-S19

55. Bjorndahl M, Cao R, Nissen LJ, Clasper S, Johnson LA, Xue Y, Zhou Z, Jackson D, Hansen AJ, Cao Y: Insulin-like growth factors 1 and 2 induce lymphangiogenesis in vivo. Proc Natl Acad Sci U S A 2005, 102:15593-15598

56. Matsuo M, Yamada S, Koizumi K, Sakurai H, Saiki I: Tumourderived fibroblast growth factor-2 exerts lymphangiogenic effects through Akt/mTOR/p70S6kinase pathway in rat lymphatic endothelial cells. Eur J Cancer 2007, 43:1748-1754

57. Cueni LN, Detmar M: New insights into the molecular control of the lymphatic vascular system and its role in disease. J Invest Dermatol 2006, 126:2167-2177

58. Szybowska P, Kostas M, Wesche J, Wiedlocha A, Haugsten EM: Cancer mutations in FGFR2 prevent a negative feedback loop mediated by the ERK1/2 pathway. Cells 2019, 8:518

59. Xie Y, Su N, Yang J, Tan Q, Huang S, Jin M, Ni Z, Zhang B, Zhang D, Luo F, Chen H, Sun X, Feng JQ, Qi H, Chen L: FGF/FGFR signaling in health and disease. Signal Transduct Target Ther 2020, 5: 181

60. Gale NW, Thurston G, Hackett SF, Renard R, Wang Q, McClain J, Martin C, Witte C, Witte MH, Jackson D, Suri C, Campochiaro PA, Wiegand SJ, Yancopoulos GD: Angiopoietin-2 is required for postnatal angiogenesis and lymphatic patterning, and only the latter role is rescued by angiopoietin-1. Dev Cell 2002, 3:411-423

61. Tammela T, Saaristo A, Lohela M, Morisada T, Tornberg J, Norrmen C, Oike Y, Pajusola K, Thurston G, Suda T, YlaHerttuala S, Alitalo K: Angiopoietin-1 promotes lymphatic sprouting and hyperplasia. Blood 2005, 105:4642-4648
62. Alitalo A, Detmar M: Interaction of tumor cells and lymphatic vessels in cancer progression. Oncogene 2012, 31:4499-4508

63. Clasper S, Royston D, Baban D, Cao Y, Ewers S, Butz S, Vestweber D, Jackson DG: A novel gene expression profile in lymphatics associated with tumor growth and nodal metastasis. Cancer Res 2008, 68:7293-7303

64. Caunt M, Mak J, Liang WC, Stawicki S, Pan Q, Tong RK, Kowalski J, Ho C, Reslan HB, Ross J, Berry L, Kasman I, Zlot C, Cheng Z, Le Couter J, Filvaroff EH, Plowman G, Peale F, French D, Carano R, Koch AW, Wu Y, Watts RJ, Tessier-Lavigne M, Bagri A: Blocking neuropilin-2 function inhibits tumor cell metastasis. Cancer Cell 2008, 13:331-342

65. Farnsworth RH, Karnezis T, Maciburko SJ, Mueller SN, Stacker SA: The interplay between lymphatic vessels and chemokines. Front Immunol 2019, 10:518

66. Fankhauser M, Broggi MAS, Potin L, Bordry N, Jeanbart L, Lund AW, Da Costa E, Hauert S, Rincon-Restrepo M, Tremblay C, Cabello E, Homicsko K, Michielin O, Hanahan D, Speiser DE, Swartz MA: Tumor lymphangiogenesis promotes $\mathrm{T}$ cell infiltration and potentiates immunotherapy in melanoma. Sci Transl Med 2017, 9:eaa14712

67. Song E, Mao T, Dong H, Boisserand LSB, Antila S, Bosenberg M, Alitalo K, Thomas JL, Iwasaki A: VEGF-C-driven lymphatic drainage enables immunosurveillance of brain tumours. Nature 2020, 577:689-694

68. Pereira ER, Jones D, Jung K, Padera TP: The lymph node microenvironment and its role in the progression of metastatic cancer. Semin Cell Dev Biol 2015, 38:98-105

69. Hirosue S, Vokali E, Raghavan VR, Rincon-Restrepo M, Lund AW, Corthesy-Henrioud P, Capotosti F, Halin Winter C, Hugues S, Swartz MA: Steady-state antigen scavenging, cross-presentation, and CD8 + T cell priming: a new role for lymphatic endothelial cells. J Immunol 2014, 192:5002-5011

70. Lane RS, Femel J, Breazeale AP, Loo CP, Thibault G, Kaempf A, Mori M, Tsujikawa T, Chang YH, Lund AW: IFNgamma-activated dermal lymphatic vessels inhibit cytotoxic $\mathrm{T}$ cells in melanoma and inflamed skin. J Exp Med 2018, 215:3057-3074

71. Lugano R, Ramachandran M, Dimberg A: Tumor angiogenesis: causes, consequences, challenges and opportunities. Cell Mol Life Sci 2020, 77:1745-1770

72. Jones D, Min W: An overview of lymphatic vessels and their emerging role in cardiovascular disease. J Cardiovasc Dis Res 2011, 2:141-152

73. Kimura T, Sugaya M, Oka T, Blauvelt A, Okochi H, Sato S: Lymphatic dysfunction attenuates tumor immunity through impaired antigen presentation. Oncotarget 2015, 6:18081-18093

74. Saif MW, Knost JA, Chiorean EG, Kambhampati SR, Yu D, Pytowski B, Qin A, Kauh JS, O'Neil BH: Phase 1 study of the antivascular endothelial growth factor receptor 3 monoclonal antibody LY3022856/IMC-3C5 in patients with advanced and refractory solid tumors and advanced colorectal cancer. Cancer Chemother Pharmacol 2016, 78:815-824

75. Gogineni A, Caunt M, Crow A, Lee CV, Fuh G, van Bruggen N, Ye W, Weimer RM: Inhibition of VEGF-C modulates distal lymphatic remodeling and secondary metastasis. PLoS One 2013, 8: e 68755

76. Karaman S, Detmar M: Mechanisms of lymphatic metastasis. J Clin Invest 2014, 124:922-928

77. Partanen TA, Alitalo K, Miettinen M: Lack of lymphatic vascular specificity of vascular endothelial growth factor receptor 3 in 185 vascular tumors. Cancer 1999, 86:2406-2412

78. Williams CS, Leek RD, Robson AM, Banerji S, Prevo R, Harris AL, Jackson DG: Absence of lymphangiogenesis and intratumoural lymph vessels in human metastatic breast cancer. J Pathol 2003, 200: 195-206

79. Alishekevitz D, Gingis-Velitski S, Kaidar-Person O, Gutter-Kapon L, Scherer SD, Raviv Z, Merquiol E, Ben-Nun Y, Miller V, Rachman- 
Tzemah C, Timaner M, Mumblat Y, Ilan N, Loven D, Hershkovitz D, Satchi-Fainaro R, Blum G, Sleeman JP, Vlodavsky I, Shaked Y: Macrophage-induced lymphangiogenesis and metastasis following paclitaxel chemotherapy is regulated by VEGFR3. Cell Rep 2016, 17: 1344-1356

80. Evans R, Flores-Borja F, Nassiri S, Miranda E, Lawler K, Grigoriadis A, Monypenny J, Gillet C, Owen J, Gordon P, Male V, Cheung A, Noor F, Barber P, Marlow R, Francesch-Domenech E, Fruhwirth G, Squadrito M, Vojnovic B, Tutt A, Festy F, De Palma M, $\mathrm{Ng} \mathrm{T}$ : Integrin-mediated macrophage adhesion promotes lymphovascular dissemination in breast cancer. Cell Rep 2019, 27: 1967-1968.e4

81. Pereira ER, Kedrin D, Seano G, Gautier O, Meijer EFJ, Jones D, Chin SM, Kitahara S, Bouta EM, Chang J, Beech E, Jeong HS, Carroll MC, Taghian AG, Padera TP: Lymph node metastases can invade local blood vessels, exit the node, and colonize distant organs in mice. Science 2018, 359:1403-1407

82. Brown M, Assen FP, Leithner A, Abe J, Schachner H, Asfour G, Bago-Horvath Z, Stein JV, Uhrin P, Sixt M, Kerjaschki D: Lymph node blood vessels provide exit routes for metastatic tumor cell dissemination in mice. Science 2018, 359:1408-1411

83. Ma L, Hernandez MO, Zhao Y, Mehta M, Tran B, Kelly M, Rae Z, Hernandez JM, Davis JL, Martin SP, Kleiner DE, Hewitt SM, Ylaya K, Wood BJ, Greten TF, Wang XW: Tumor cell biodiversity drives microenvironmental reprogramming in liver cancer. Cancer Cell 2019, 36:418-430.e6

84. Faivre S, de Gramont A, Raymond E: Learning from 7 years of experience with sorafenib in advanced HCC: sorafenib better than sorafenib? Target Oncol 2016, 11:565-567

85. Cho YK, Shin EY, Uehara H, Ambati BK: Effect of sorafenib in a murine high risk penetrating keratoplasty model. Int J Ophthalmol 2017, 10:834-839

86. Nair A, Reece K, Donoghue MB, Yuan WV, Rodriguez L, Keegan P, Pazdur R: FDA supplemental approval summary: lenvatinib for the treatment of unresectable hepatocellular carcinoma. Oncologist 2021, 26:e484-e491

87. Matsui J, Funahashi Y, Uenaka T, Watanabe T, Tsuruoka A, Asada M: Multi-kinase inhibitor E7080 suppresses lymph node and lung metastases of human mammary breast tumor MDA-MB-231 via inhibition of vascular endothelial growth factor-receptor (VEGF-R) 2 and VEGF-R3 kinase. Clin Cancer Res 2008, 14:5459-5465

88. Yamamoto Y, Matsui J, Matsushima T, Obaishi H, Miyazaki K, Nakamura K, Tohyama O, Semba T, Yamaguchi A, Hoshi SS, Mimura F, Haneda T, Fukuda Y, Kamata JI, Takahashi K, Matsukura M, Wakabayashi T, Asada M, Nomoto KI, Watanabe T, Dezso Z, Yoshimatsu K, Funahashi Y, Tsuruoka A: Lenvatinib, an angiogenesis inhibitor targeting VEGFR/FGFR, shows broad antitumor activity in human tumor xenograft models associated with microvessel density and pericyte coverage. Vasc Cell 2014, 6:18
89. He Y, Rajantie I, Pajusola K, Jeltsch M, Holopainen T, YlaHerttuala S, Harding T, Jooss K, Takahashi T, Alitalo K: Vascular endothelial cell growth factor receptor 3-mediated activation of lymphatic endothelium is crucial for tumor cell entry and spread via lymphatic vessels. Cancer Res 2005, 65:4739-4746

90. He Y, Kozaki K, Karpanen T, Koshikawa K, Yla-Herttuala S, Takahashi T, Alitalo K: Suppression of tumor lymphangiogenesis and lymph node metastasis by blocking vascular endothelial growth factor receptor 3 signaling. J Natl Cancer Inst 2002, 94:819-825

91. Mita AC, Takimoto CH, Mita M, Tolcher A, Sankhala K, Sarantopoulos J, Valdivieso M, Wood L, Rasmussen E, Sun YN, Zhong ZD, Bass MB, Le N, LoRusso P: Phase 1 study of AMG 386, a selective angiopoietin 1/2-neutralizing peptibody, in combination with chemotherapy in adults with advanced solid tumors. Clin Cancer Res 2010, 16:3044-3056

92. Spratlin JL, Cohen RB, Eadens M, Gore L, Camidge DR, Diab S, Leong S, O'Bryant C, Chow LQ, Serkova NJ, Meropol NJ, Lewis NL, Chiorean EG, Fox F, Youssoufian H, Rowinsky EK, Eckhardt SG: Phase I pharmacologic and biologic study of ramucirumab (IMC-1121B), a fully human immunoglobulin G1 monoclonal antibody targeting the vascular endothelial growth factor receptor-2. J Clin Oncol 2010, 28:780-787

93. Zou H, Wang F, Zhou JJ, Liu X, He Q, Wang C, Zheng YW, Wen Y, Xiong L: Application of photodynamic therapy for liver malignancies. J Gastrointest Oncol 2020, 11:431-442

94. Tammela T, Saaristo A, Holopainen T, Yla-Herttuala S, Andersson LC, Virolainen S, Immonen I, Alitalo K: Photodynamic ablation of lymphatic vessels and intralymphatic cancer cells prevents metastasis. Sci Transl Med 2011, 3:69ra11

95. Kilarski WW, Muchowicz A, Wachowska M, Mezyk-Kopec R, Golab J, Swartz MA, Nowak-Sliwinska P: Optimization and regeneration kinetics of lymphatic-specific photodynamic therapy in the mouse dermis. Angiogenesis 2014, 17:347-357

96. Sato K, Zhang W, Safarikia S, Isidan A, Chen AM, Li P, Francis H, Kennedy L, Baiocchi L, Alvaro D, Glaser S, Ekser B, Alpini G: Organoids and spheroids as models for studying cholestatic liver injury and cholangiocarcinoma. Hepatology 2020, 74:491-502

97. Marin JJG, Herraez E, Lozano E, Macias RIR, Briz O: Models for understanding resistance to chemotherapy in liver cancer. Cancers (Basel) 2019, 11:1677

98. Zeng H, Liu G, Rea PA, Kruh GD: Transport of amphipathic anions by human multidrug resistance protein 3. Cancer Res 2000, 60: 4779-4784

99. Glavinas H, Mehn D, Jani M, Oosterhuis B, Heredi-Szabo K, Krajcsi P: Utilization of membrane vesicle preparations to study drug- $\mathrm{ABC}$ transporter interactions. Expert Opin Drug Metab Toxicol 2008, 4:721-732

100. Marin JJG, Lozano E, Briz O, Al-Abdulla R, Serrano MA, Macias RIR: Molecular bases of chemoresistance in cholangiocarcinoma. Curr Drug Targets 2017, 18:889-900 